\title{
SARS-CoV-2 immunochromatographic IgM/IgG rapid test in pregnancy: A false friend?
}

\author{
M Fabre' (D, S Ruiz-Martinez ${ }^{2,3}$, ME Monserrat Cantera ${ }^{2}$, A Cortizo Garrido², \\ Z Beunza Fabra', M Peran', R Benito ${ }^{4}$, P Mateo $^{2}$, C Paules $^{2,3}$ and D Oros ${ }^{2,3}$
}

\begin{abstract}
Background: An increasing body of evidence has revealed that SARS-CoV-2 infection in pregnant women could increase the risk of adverse maternal and fetal outcomes. Careful monitoring of pregnancies with COVID-I9 and measures to prevent neonatal infection are warranted. Therefore, rapid antibody tests have been suggested as an efficient screening tool during pregnancy.

Cases: We analysed the clinical performance during pregnancy of a rapid, lateral-flow immunochromatographic assay for qualitative detection of SARS-CoV-2 IgG/lgM antibodies. We performed a universal screening including 169 patients during their last trimester of pregnancy. We present a series of 14 patients with positive SARS-CoV-2 immunochromatographic assay rapid test result. Immunochromatographic assay results were always confirmed by chemiluminescent microparticle immunoassays for quantitative detection of SARS-CoV-2 IgG and IgM+lgA antibodies as the gold standard. We observed a positive predictive value of $50 \%$ and a false positive rate of $50 \%$ in pregnant women, involving a significantly lower diagnostic performance than reported in non-pregnant patients.

Discussion: Our data suggest that although immunochromatographic assay rapid tests may be a fast and profitable screening tool for SARS-CoV-2 infection, they may have a high false positive rate and low positive predictive value in pregnant women. Therefore, immunochromatographic assay for qualitative detection of SARS-CoV-2 IgG/lgM antibodies must be verified by other test in pregnant patients.
\end{abstract}

\section{Keywords}

Clinical studies, immunoassay, laboratory methods, pregnancy

Accepted: 18th November 2020

\section{Introduction}

Pregnant women and their fetuses represent a high-risk population during infectious disease outbreaks. Immunological changes during pregnancy may affect the risk of developing severe complications in COVID-19 patients. ${ }^{1}$ Although the majority of mothers do not develop any major complications, an increasing body of evidence revealed that SARSCoV-2 infection in pregnant women can cause miscarriage, severe maternal morbidity and preterm delivery.
'Aragon Institute of Health Research (IIS Aragon) Biochemistry Department, Hospital Clínico Universitario Lozano Blesa, Zaragoza, Spain

${ }^{2}$ Aragon Institute of Health Research (IIS Aragon), Obstetrics Department, Hospital Clínico Universitario Lozano Blesa, Zaragoza, Spain

${ }^{3}$ Red de Salud Materno Infantil y del Desarrollo (SAMID), RETICS. Instituto de Salud Carlos III (ISCIII), Subdirección General de Evaluación y Fomento de la Investigación y Fondo Europeo de Desarrollo Regional (FEDER) Ref: RDI6/0022/0013

${ }^{4}$ Microbiology Department, Hospital Clínico Universitario Lozano Blesa, Zaragoza, Spain. Universidad de Zaragoza. IIS Aragón. CIBERehd

\section{Corresponding author:}

S Ruiz-Martinez, Obstetric Department, Hospital Clínico Universitario Lozano Blesa, Avenida San Juan Bosco, I5., Zaragoza 50009, Spain.

Email: sruizmart@gmail.com 
Nevertheless, there is still considerable controversy about the impact of SARS-CoV-2 infection on pregnant women and the fetus. Recent studies report SARS-CoV-2 viral particles in breast milk, placental infection with the associated inflammatory changes demonstrated by immunohistochemistry and high viral load, and following by neonatal viremia. ${ }^{2}$

Careful monitoring of pregnancies with COVID-19 and measures to prevent neonatal infection are warranted. Some authors recommend universal screening for SARS-CoV-2 infection in pregnant women at the time of delivery or during pregnancy. Currently, the reverse-transcription polymerase chain reaction (RT-PCR) on respiratory samples are the gold standard for the diagnosis of active SARS-CoV-2 infection. Unfortunately, RT-PCR is time-consuming and requires specialized operators. However, easy to perform, fast and low-cost immunochromatographic $\mathrm{IgM} / \mathrm{IgG}$ rapid antibody tests have been suggested as an efficient screening tool during pregnancy.

\section{Materials and methods}

We analysed the clinical performance during pregnancy of a lateral-flow immunochromatographic assay (ICA) for qualitative detection of SARS-CoV-2 $\mathrm{IgG} / \mathrm{IgM}$ antibodies (Wondfo SARS-CoV-2 Antibody Test, Guangzhou Wondfo Biotech Co., Guangzhou, China). Between April 27 and May 29 2020, we performed a universal screening including 169 pregnant women who were either at 36 weeks of gestation or had COVID-19 symptoms in the third trimester. ICA was performed according to manufacturer's instruction, was carried out by a healthy care professional, the fingerstick whole blood specimen has been applied to the test by hanging drops and any band intensity was considered as a positive result. Fourteen patients had positive ICA results. Almost half of them (57.1\%) reported having presented in the previous weeks mildsymptoms (Table 1). Active infection was ruled out by negative RT-PCR results (Abbott RealTime SARSCoV-2 Assay, Abbott Molecular, Abbott Park, IL, USA) from nasopharyngeal swabs. ICA results were also confirmed by chemiluminescent microparticle immunoassays (CMIA) for quantitative detection of SARS-CoV-2 IgG (IgG-CMIA; SARS-CoV-2 IgG Assay, Abbott Laboratories Ireland, Dublin, Ireland) and IgM+IgA antibodies (IgM+IgA-CLIA; COVID19 VIRCLIA $\operatorname{IgM}+\operatorname{IgA}$, Vircell Microbiologists, Granada, Spain) as the gold standard. ${ }^{3}$ The maternal blood was taken with vacutainer in a tube without anticoagulant and has been allowed to clot. The samples were centrifuged and were determined on the same day. A signal of-cut-off $(\mathrm{S} / \mathrm{CO})$ index $>1.4$ were considered positive for IgG. IgM+IgA samples with a $\mathrm{S} / \mathrm{CO}$ index less than 0.4 were considered negative samples, those with a $\mathrm{S} / \mathrm{CO}$ index more than 0.6 were considered positive and samples with a $\mathrm{S} / \mathrm{CO}$ ranging from 0.4 to 0.6 were interpreted as grey zone.

\section{Results}

According to the gold standard results, a total of 7 out of $14(50 \%)$ patients were considered true positive ICA tests. Six patients were directly positive for IgG-CMIA. Additionally, one case (number 10) initially presented an uncertain result for $\operatorname{IgM}+\operatorname{IgA}$-CLIA with negative IgG-CLIA, hence she was considered as recent infection, as two weeks later she finally had SARS-CoV-2 positive IgG-CLIA (Table 1). The remaining seven patients tested negative for both IgG-CMIA and IgM+IgA-CLIA, and therefore were considered ICA false-positive results. It is important to note that, in contrast with the previously described case number 10 , cases number 6 and number 12 were considered as false-positive ICA results, as after being initially positive for $\operatorname{IgM}+\operatorname{IgA}-\mathrm{CLIA}$ (S/Co index 1.1 and 1.09 , respectively) but negative for IgG-CMIA, both IgM+IgA-CLIA and IgG-CMIA assays were negative two weeks later (Table 1). Recent studies suggest that humoral immunity against SARS-CoV-2 may not be long lasting in persons with mild illness. We evaluated this possibility in cases number 6 and number 12, as in both patients levels of $\operatorname{IgM}+\operatorname{IgA}$ and $\operatorname{IgG}$ decreased between test. However, they never presented positive results for IgG-CMIA or any symptoms or signs of SARS-CoV-2 infection.

\section{Discussion}

Although since the beginning of this pandemic RTPCR has been used to confirm diagnosis of SARS$\mathrm{CoV}-2$, serological assays can play an important role in the management of virus infection. Serologic tests detect waning or past SARS-CoV-2 virus infection indirectly by measuring the host humoral immune response to the virus. A meta-analysis of diagnostic performance of serological test in general population yielded a sensitivity of $82 \%(95 \% \mathrm{CI}: 75-88 \%)$ for IgM, and $85 \%$ for $\operatorname{IgG}(95 \% \mathrm{CI}: 73-93 \%){ }^{4}$. The fact that both RT-PCR and serologic assays, need to be determinate by a clinical laboratory, make interesting the evaluation of the point of care testing as the ICA rapid test.

It should be noted that as pandemic progresses, the value of diagnostic testing for SARS-CoV-2 has enhanced. We considered our available technology as gold standard but virus neutralization remains the gold standard for determining antibody efficacy. Although, this is currently not available. For future studies, it 


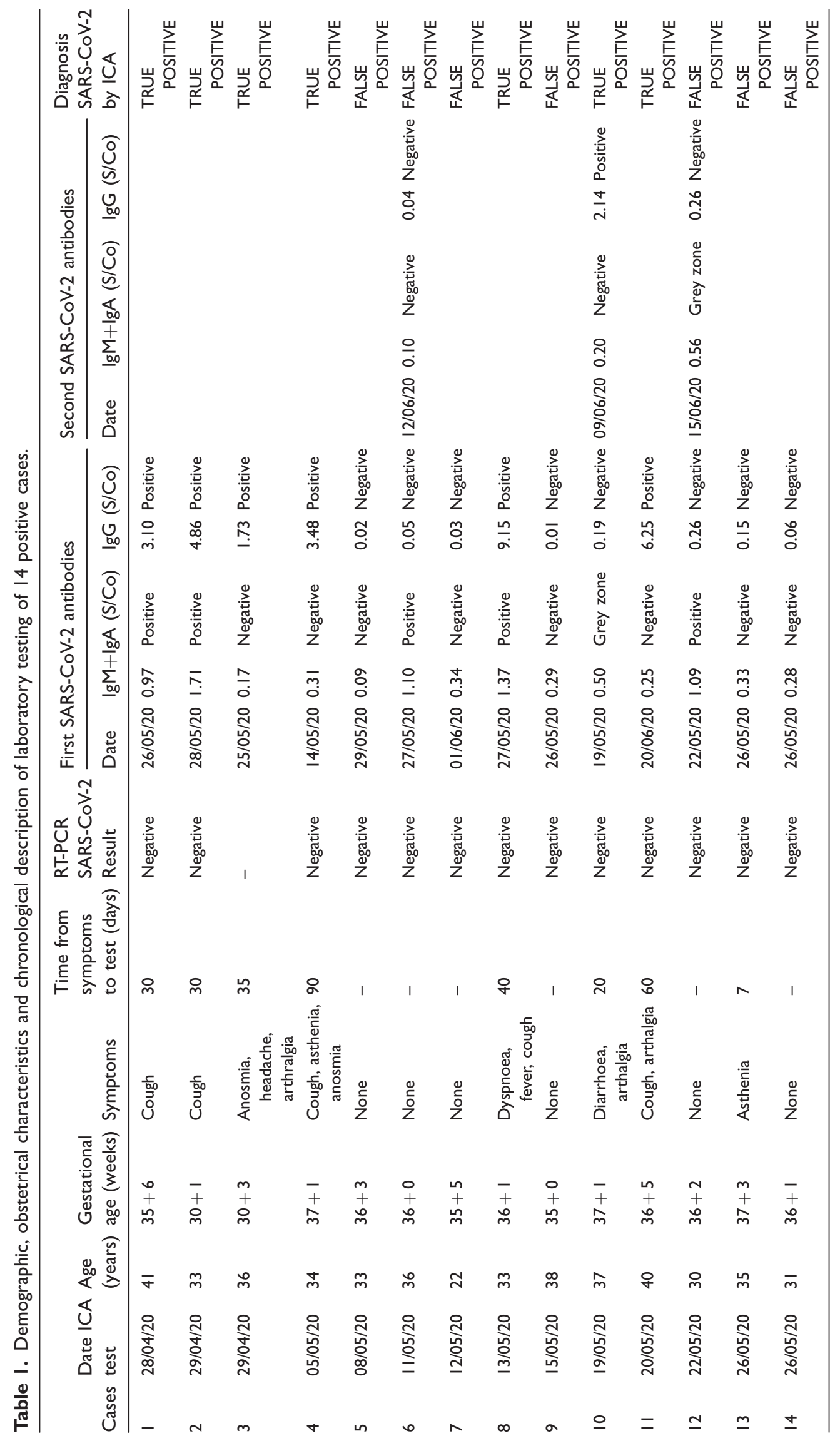


would be helpful to compare different immunoassay for SARS-CoV-2 antibodies in pregnant population.

Newly, lateral-flow immunochromatographic assay in non-pregnant patients has shown a moderate sensitivity $(84.4 \%)$ and high specificity $(98.6 \%))^{5}$ In contrast, we observed in our series of cases a positive predictive value of $50 \%$ with a false positive rate of $50 \%$ in pregnant women. Unfortunately, we could not evaluate the false-negative rate in our study. However, false-positive results may have a detrimental effect on pregnant patients, such as false security awareness increasing the risk of infection, unnecessary treatment, anxiety or depression.

A false-positive result by ICA test in pregnant women may be explained by several factors. Rheumatoid factors, antinuclear and heterophile antibodies are known to interfere with rapid antibody tests. Moreover, cross-react with various agents such as influenza-A, respiratory syncytial virus or other coronaviruses have been reported. ${ }^{6}$

The complexity of the immunology of pregnancy makes it hard to interpret the test results. If there is any clinical suspicion of discordance between the clinical and the laboratory data, an attempt should be made to resolve the difference. The CMIA can minimize these interferences by careful choices of reagents, dilution, depletion or blocking, while rapid antibody tests cannot.

It is important to emphasize that the false positive rate depends on the prevalence of disease in the population. Instead, the sensitivity and specificity of the diagnostics test are independent of the prevalence. Thus, decisions made in low prevalence settings must not be automatically extrapolated to settings with high disease prevalence.

This study has several limitations. The first limitation is the small number of participants and the inability to confirm all ICA test by CMIA. It would have been interesting to perform ICA test and CMIA test on the same sample or at least on the same type of sample. In fact, recently it has been demonstrated significantly superior sensitivity with serum $(80 \%)$ than with whole blood $(57 \%)$ in ICA test. ${ }^{5}$ However, while serum show better results than whole blood, serum samples have the disadvantage of requiring additional laboratory material. Despite these constrains, our study is designed to be a tool for others professionals in clinical practice.

To our knowledge, this is the first population-based study to evaluate a SARS-CoV-2 rapid antibody test in pregnant women. Our data suggest that although immunochromatographic assay for qualitative detection of SARS-CoV-2 IgG/IgM antibodies may be a fast and profitable screening tool, they have a high false positive rate in pregnant women. Thus, ICA antibodies SARS-CoV-2 tests must be verified by other test methods such as chemiluminescent microparticle immunoassays in pregnant patients.

\section{Acknowledgements}

S Ruiz-Martinez and C Paules were supported by a research grant from the Instituto de Salud Carlos III (CM18/00202, JR19/00006).

\section{Declaration of conflicting interests}

The author(s) declared no potential conflicts of interest with respect to the research, authorship, and/or publication of this article.

\section{Funding}

The author(s) received no financial support for the research, authorship, and/ or publication of this article.

\section{Ethical approval}

This study was approved by the Hospital Clinico Lozano Blesa.

\section{Guarantor}

SRM.

\section{Contributorship}

MF and MP have analysed the diagnostic test, SRM, PM, CP and DO have collected and analysed the clinical and demographic data of each patient, MF, SRM, RB and DO have written the manuscript. All authors reviewed and edited the manuscript and approved the final version of the manuscript.

\section{ORCID iD}

M Fabre (iD https://orcid.org/0000-0003-4744-0903

\section{References}

1. Sarapultsev A and Sarapultsev P. Immunological environment shifts during pregnancy may affect the risk of developing severe complications in COVID-19 patients. Am J Reprod Immunol 2020; 84: e13285.

2. Vivanti AJ, Vauloup-Fellous $\mathrm{C}$, Prevot $\mathrm{S}$, et al. Transplacental transmission of SARS-CoV-2 infection. Nat Commun 2020; 11: 3572.

3. Bryan A, Pepper G, Wener MH, et al. Performance characteristics of the Abbott architect SARS-CoV-2 IgG assay and seroprevalence in Boise, Idaho. J Clin Microbiol 2020; 58: e00941-20.

4. Caini S, Bellerba F, Corso F, et al. Meta-analysis of diagnostic performance of serological tests for SARS-CoV-2 antibodies up to 25 April 2020 and public health implications. Euro Surveill 2020; 25: 2000980.

5. Flower B, Brown JC, Simmons B, et al. Clinical and laboratory evaluation of SARS-CoV-2 lateral flow assays for use in a national COVID-19 seroprevalence survey. Thorax 2020; 75: 1082-1088.

6. Okba NMA, Müller MA, Li W, et al. Severe acute respiratory syndrome coronavirus 2-specific antibody responses in coronavirus disease patients. Emerg Infect Dis 2020; 26: 1478-1488. 